\title{
Rewiring yeast acetate metabolism through MPC1 loss of function leads to mitochondrial damage and decreases chronological lifespan
}

\author{
Ivan Orlandii, ${ }^{1,2}$ Damiano Pellegrino Coppola ${ }^{2}$ and Marina Vai ${ }^{1,2, *}$ \\ ${ }^{1}$ SYSBIO Centre for Systems Biology Milano, Italy. \\ 2 Dipartimento di Biotecnologie e Bioscienze, Università di Milano-Bicocca, Piazza della Scienza 2, 20126 Milano, Italy. \\ * Corresponding Author: Marina Vai, Dipartimento di Biotecnologie e Bioscienze, Università di Milano-Bicocca, Piazza della Scienza 2; \\ 20126 Milano, Italy; Tel: +39 0264483531; Fax: +39 0264483565; Email: marina.vai@unimib.it
}

\begin{abstract}
During growth on fermentable substrates, such as glucose, pyruvate, which is the end-product of glycolysis, can be used to generate acetylCoA in the cytosol via acetaldehyde and acetate, or in mitochondria by direct oxidative decarboxylation. In the latter case, the mitochondrial pyruvate carrier (MPC) is responsible for pyruvate transport into mitochondrial matrix space. During chronological aging, yeast cells which lack the major structural subunit Mpc1 display a reduced lifespan accompanied by an age-dependent loss of autophagy. Here, we show that the impairment of pyruvate import into mitochondria linked to Mpc1 loss is compensated by a flux redirection of TCA cycle intermediates through the malic enzyme-dependent alternative route. In such a way, the TCA cycle operates in a "branched" fashion to generate pyruvate and is depleted of intermediates. Mutant cells cope with this depletion by increasing the activity of glyoxylate cycle and of the pathway which provides the nucleocytosolic acetyl-CoA. Moreover, cellular respiration decreases and ROS accumulate in the mitochondria which, in turn, undergo severe damage. These acquired traits in concert with the reduced autophagy restrict cell survival of the mpc1s mutant during chronological aging. Conversely, the activation of the carnitine shuttle by supplying acetyl-CoA to the mitochondria is sufficient to abrogate the short-lived phenotype of the mutant.
\end{abstract}

\author{
doi: $10.15698 / \operatorname{mic} 2014.12 .178$ \\ Received originally: 28.06 .2014 ; \\ in revised form: 21.10.2014, \\ Accepted 05.11.2014, \\ Published 18.11.2014. \\ Keywords: acetyl-COA, chronological \\ aging, Mcp1, mitochondria, pyruvate, \\ Saccharomyces cerevisiae. \\ Abbreviations: \\ Ach1 - acetyl-CoA hydrolase 1, \\ Acs - acetyl-COA synthetase, \\ Adh - alcohol dehydrogenase, \\ Ald - aldehyde dehydrogenase, \\ CLS - chronological lifespan, \\ DW - cell dry weight, \\ Icl1 - isocitrate lyase, \\ Mae1 - malic enzyme \\ MPC - mitochondrial pyruvate carrier, \\ Pck1 - phosphoenolpyruvate \\ carboxykinase 1, \\ TCA cycle - tricarboxylic acid cycle, \\ $w t$ - wild type.
}

\section{INTRODUCTION}

Aging of postmitotic quiescent mammalian cells has been modelled in the yeast Saccharomyces cerevisiae by its chronological lifespan (CLS) [1, 2]. CLS represents the length of time a culture of nondividing cells remains viable in stationary phase: viability is assessed by the ability to resume growth upon return to rich medium [3]. Evidence to date indicates that chronological aging is intimately regulated by signaling pathways which sense nutrient availability, namely TORC1-Sch9 and Ras-PKA, and carbon metabolism [4, 5]. In this context, emerging data on some metabolites and nutrient manipulation/dietary regimens which proved to modulate aging not only in yeast but also in evolutionary diverse organisms have opened up new opportunities for therapeutic interventions promoting healthy aging in humans $[6,7]$. In particular, among the main metabolic intermediates, acetyl-CoA is increasingly being acknowledged as an important regulator of longevity [8-10]. This metabolite is the activated form of acetate obtained via a thioester linkage with coenzyme A which cells use for macromolecule biosynthesis. Furthermore, in the mitochondria it is a crucial substrate for energy production since it fuels the TCA cycle and consequently the production of reducing equivalents which enter the electron transport chain and support the oxidative phosphorylation. In addition, acetyl-CoA also supplies the acetyl group for protein acetylation, a dynamic posttranslational modification which occurs on a wide range of substrates, including histones and many metabolic enzymes, thus connecting metabolism, epigenetics and 
transcriptional regulation [11-14]. Recently, by manipulating the major routes of acetyl-CoA formation in yeast and mammalian cells, it has been shown that the nucleocytosolic pool of acetyl-CoA acts as a dominant suppressor of cytoprotective autophagy during aging [8, 10]. In line with this, during aging, histone hypoacetylation correlates with enhanced expression of ATG genes and induction of autophagy [15]. This is a degradative process which is crucial for the maintenance of cellular homeostasis by removing misfolded/damaged or "obsolete" proteins and organelles, including mitochondria. It becomes fundamental in nondividing cells where the intracellular damage cannot be "diluted" [16]. Nutrient depletion and inactivations of genes in the central nutrient signaling pathways are known inducers of autophagy [17].

In S.cerevisiae, the nucleocytosolic pool of acetyl-CoA is synthesized by the acetyl-CoA synthetase 2 (Acs2) by activation of acetate in an ATP-dependent reaction. This enzyme is known as the glycolytic isoform [18] and besides its role in carbon metabolism it is required for histone acetylation [19]. The mitochondrial acetyl-CoA pool is generated by the Acs1 (the gluconeogenic isoform) and by the acetyl-CoA hydrolase 1 (Ach1) which catalyzes the transfer of the CoASH moiety from succinyl-CoA to acetate
[20]. Moreover, according to culture conditions, acetyl-CoA can be formed and utilized in different ways. During growth on fermentable substrates, such as glucose, it is generated from pyruvate. This compound is the end-product of glycolysis and is a key node in the branching point between respiratory metabolism and alcoholic fermentation as well as assimilatory and dissimilatory metabolic reactions [21]. At the branching point, it can follow three major fates (Fig. 1): (i) decarboxylation to acetaldehyde which generates acetyl-CoA by the pyruvate dehydrogenase (PDH) bypass; (ii) anaplerotic carboxylation to oxaloacetate and (iii) the direct oxidative decarboxylation to acetyl-CoA by the PDH complex, which is located in the mitochondrial matrix. Pyruvate can cross the outer mitochondrial membrane while the passage across the inner mitochondrial membrane requires the mitochondrial pyruvate carrier (MPC) $[22,23]$. This carrier effectively represents a link between cytosolic pyruvate metabolism and the TCA cycle. Loss of the major structural subunit $\mathrm{Mpc1}$ results in defective mitochondrial pyruvate uptake [22] and, during chronological aging, in a short-lived phenotype accompanied by an age-dependent loss of autophagy [8].

In this work we investigated the metabolic changes underlying MPC1 loss of function. We found that $m p c 1 \Delta$ cells

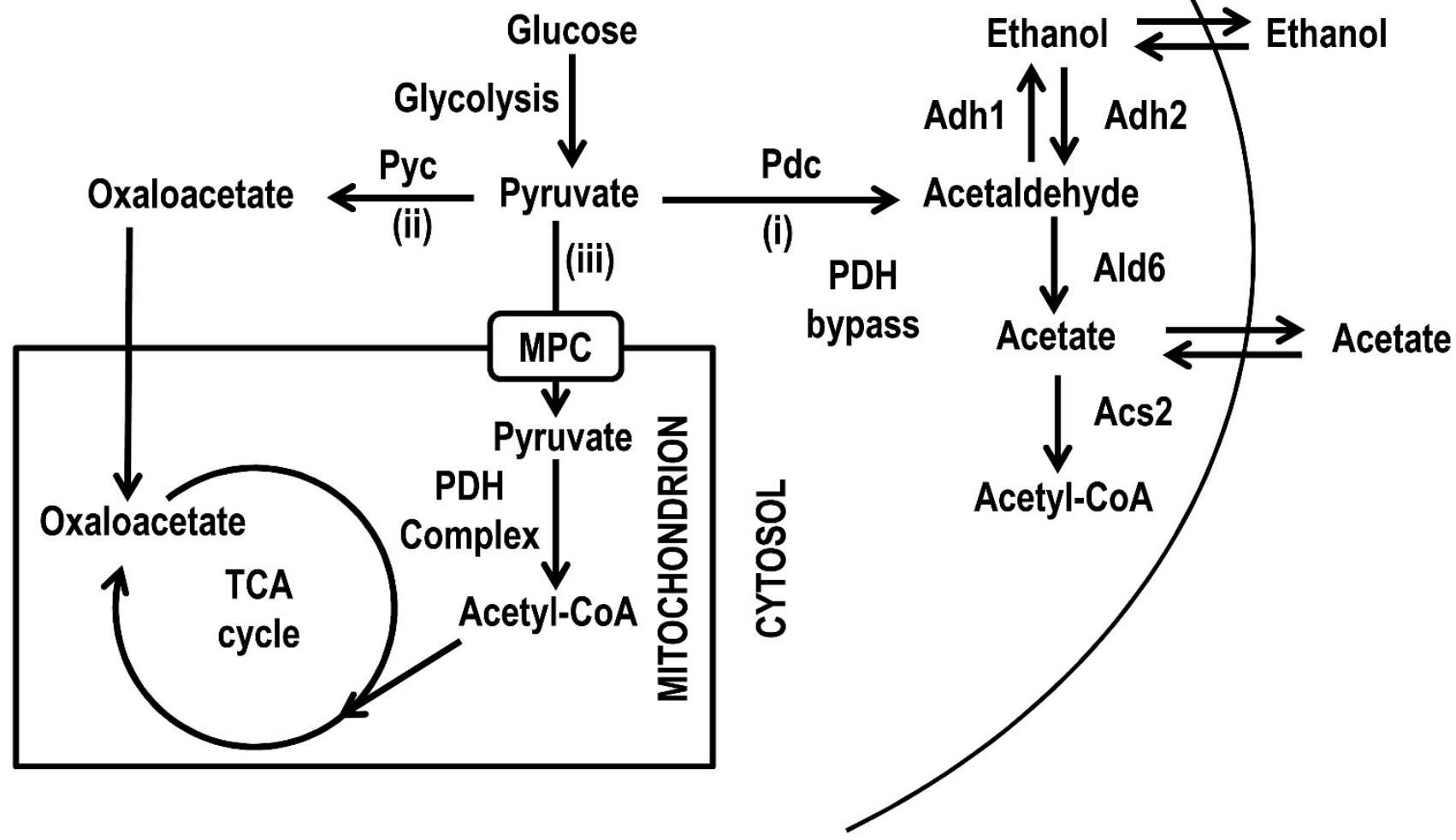

FIGURE 1: Scheme of metabolic pathways allowing pyruvate utilization. The three pathways which originate from pyruvate after (i) decarboxylation to acetaldehyde, (ii) carboxylation to oxaloacetate and (iii) oxidative decarboxylation to acetyl-CoA are schematically shown. Acs, acetyl-CoA synthase; Adh, alcohol dehydrogenase; Ald, aldehyde dehydrogenase; MPC, mitochondrial pyruvate carrier; Pdc, pyruvate decarboxylase; PDH, pyruvate dehydrogenase; Pyc, pyruvate carboxylase. 
make up for their impairment in mitochondrial pyruvate with a metabolic rewiring which involves several intermediates of the mitochondrially localized TCA cycle and the cytosolic glyoxylate shunt but ultimately results in a proaging process.

\section{RESULTS AND DISCUSSION}

Lack of Mpc1 is accompanied by an increase of Ald enzymatic activities

Since an impairment in the import of pyruvate into mitochondria linked to MPC1 deletion significantly restricted CLS (Fig. 2A) [8], we decided to analyze in more detail the metabolic changes underlying this short-lived phenotype. Initially, in the context of a standard CLS experiment [3],
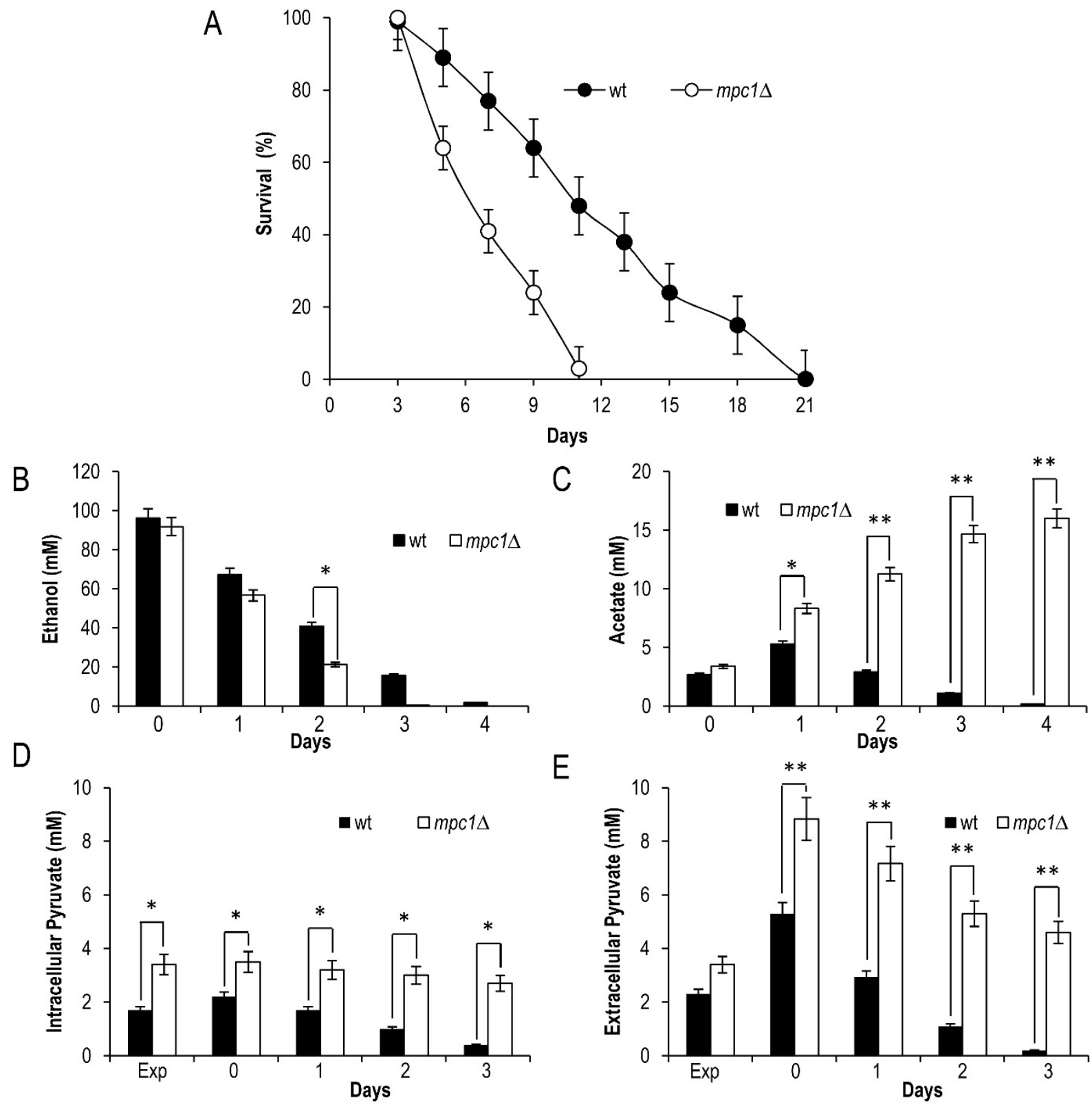

E

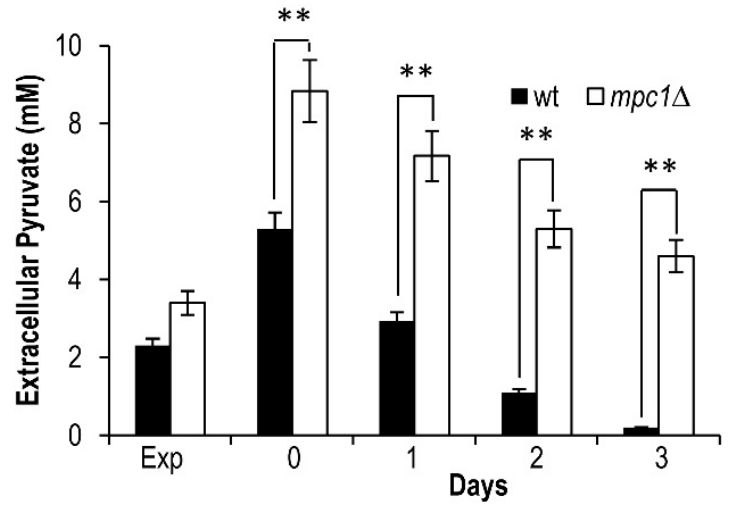

FIGURE 2: MPC1 inactivation shortens CLS in concert with increased extracellular acetate and pyruvate. Wild type (wt) and $m p c 1 \Delta$ mutant cells were grown in minimal medium/2\% glucose and the required supplements in excess (see Materials and Methods) and followed up to stationary phase. (A) CLS of wt and $m p c 1 \Delta$ mutant cells. At each time-point, survival was determined by colony-forming capacity. $72 \mathrm{~h}$ after the diauxic shift (Day 3) was considered the first age-point (see Materials and Methods). Day 0, diauxic shift. Data refer to mean values of three independent experiments. Standard deviations (SD) are indicated. Bar charts of extracellular ethanol (B) and acetate (C) concentrations at different time points after the diauxic shift (Day 0). In parallel, intracellular (D) and extracellular (E) pyruvate concentrations were measured. Exp, exponential growth phase. Data refer to mean values of three independent experiments. SD is indicated. Statistical significance as assessed by one-way ANOVA test is indicated ( ${ }^{*} \mathrm{P} \leq 0.05$ and $\left.{ }^{* *} \mathrm{P} \leq 0.01\right)$. 
we measured the levels of some metabolites such as pyruvate, ethanol and acetate. These last two compounds are produced during glucose fermentation following decarboxylation of cytosolic pyruvate to acetaldehyde by pyruvate decarboxylase (Pdc) (Fig. 1). Only upon glucose depletion, does the diauxic shift occurs and yeast cells switch to a respiration-based metabolism of the fermentation $\mathrm{C} 2$ byproducts. Finally, when these carbon/energy sources are fully exhausted, cells enter a quiescent stationary phase. At the diauxic shift, in the mpc1 $\Delta$ culture the amount of ethanol and acetate was similar to that in the wild type (wt) culture (Fig. S1, 2B and C). Differently, during the postdiauxic phase, in the mutant the consumption of ethanol, which is re-introduced into the metabolism via its oxidation to acetate (Fig. 1), was not affected significantly compared to that in the wt (specific consumption rate, qEtOH, of $1.43 \pm 0.04 \mathrm{mmol} \cdot \mathrm{g} \cdot \mathrm{DW}^{-1} \cdot \mathrm{h}^{-1}$ for the mutant and $1.12 \pm$ $0.06 \mathrm{mmol} \cdot \mathrm{g} \cdot \mathrm{DW}^{-1} \cdot \mathrm{h}^{-1}$ for the wt) (Fig. S1 and $2 \mathrm{~B}$ ), while the acetate continued to accumulate in the medium (Fig. 2C). Such a prolonged secretion of acetate throughout the ethanol consumption phase suggests that in the mpc1s mutant there is an imbalance between acetate production rate from acetaldehyde and its conversion rate into acetylCoA. In fact, the acetate transport relies on an active transport for the dissociated form of the acid (subjected to glucose repression) accompanied by passive/facilitated diffusion of the undissociated acid [24]. During the post- diauxic phase the $\mathrm{pH}$ of the medium is far below the pKa of acetic acid (4.75) [25] and according to the HendersonHasselbalch equation, acetic acid is substantially undissociated: $98.6 \%$ at pH 2.9 (the value we measured at Day 3 after the diauxic shift). Consequently, in a condition where transmembrane diffusion strongly prevails over the active transport, the acetate export/import will take place according to the gradient between the intracellular and extracellular concentrations of acetate.

Concerning intracellular pyruvate, its concentration was higher in the mpc1 $\Delta$ mutant compared to that in the wt not only in exponential phase, as already observed by [22], but also at/after the diauxic shift (Fig. 2D). This was also associated with an increase in the extracellular pyruvate (Fig. 2E) which reflects an overflow of pyruvate within the cytosol. Similar results were obtained when the experiments were also performed by growing the histidineprototroph mpc1 $1 \Delta$ mutant (mcp1 $1:: H I S 3)$ in a histidinesupplemented medium as previously carried out for the wt (Fig. S2) indicating that the different composition of amino acids in the medium does not affect the results.

Afterwards, we measured the enzymatic activities of alcohol dehydrogenases (Adhs) catalysing the interconversion of acetaldehyde and ethanol [26] and of acetaldehyde dehydrogenases (Alds) which produce acetate by oxidizing the acetaldehyde generated from pyruvate during fermentation and that obtained during ethanol oxidation (Fig. 1).
A

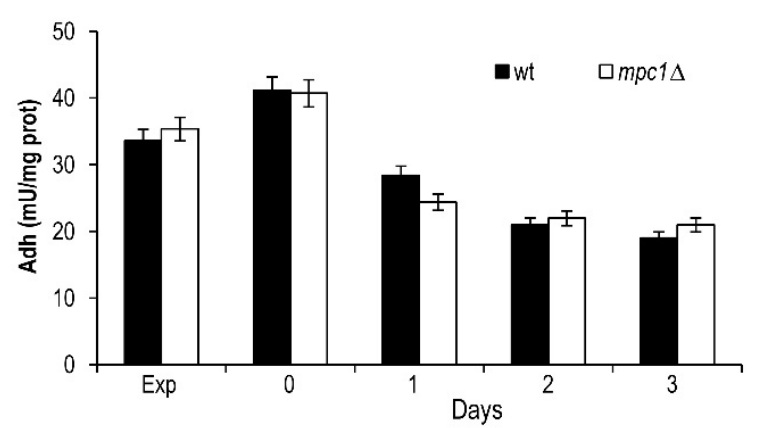

C

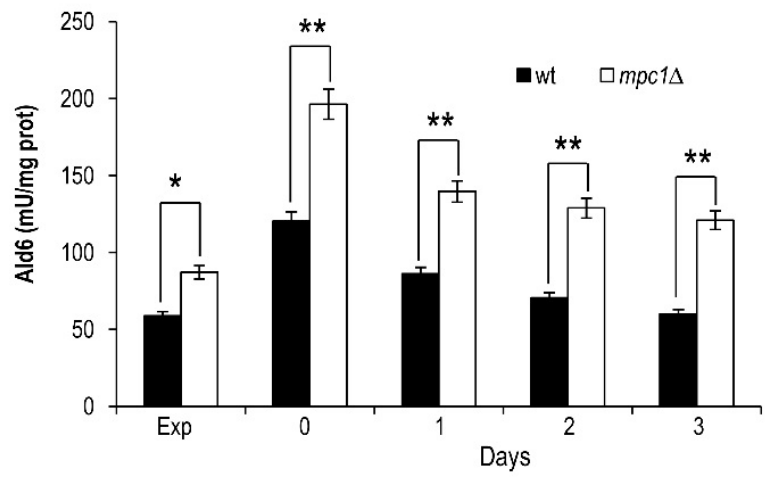

B
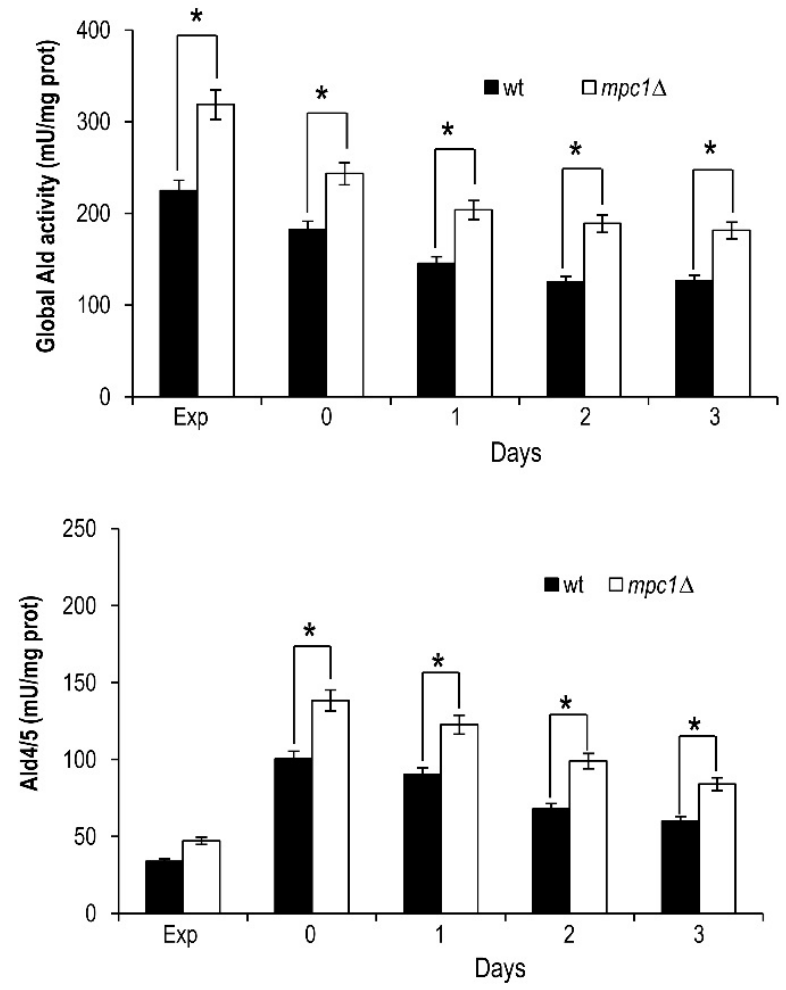

FIGURE 3: In mpc1s cells the extracellular abundance of acetate correlates with enhanced Ald enzymatic activity. Bar charts of total Adh (A), total Ald (B), Ald6 (C) and Ald4/5 (D) enzymatic activities measured at the indicated time points for wt and $m p c 1 \Delta$ mutant cells grown as in Figure 2. Exp, exponential growth phase. Day 0, diauxic shift. Data refer to mean values determined in three independent experiments. SD is indicated. $* \mathrm{P} \leq 0.05$ and $* * \mathrm{P} \leq 0.01$. 
A
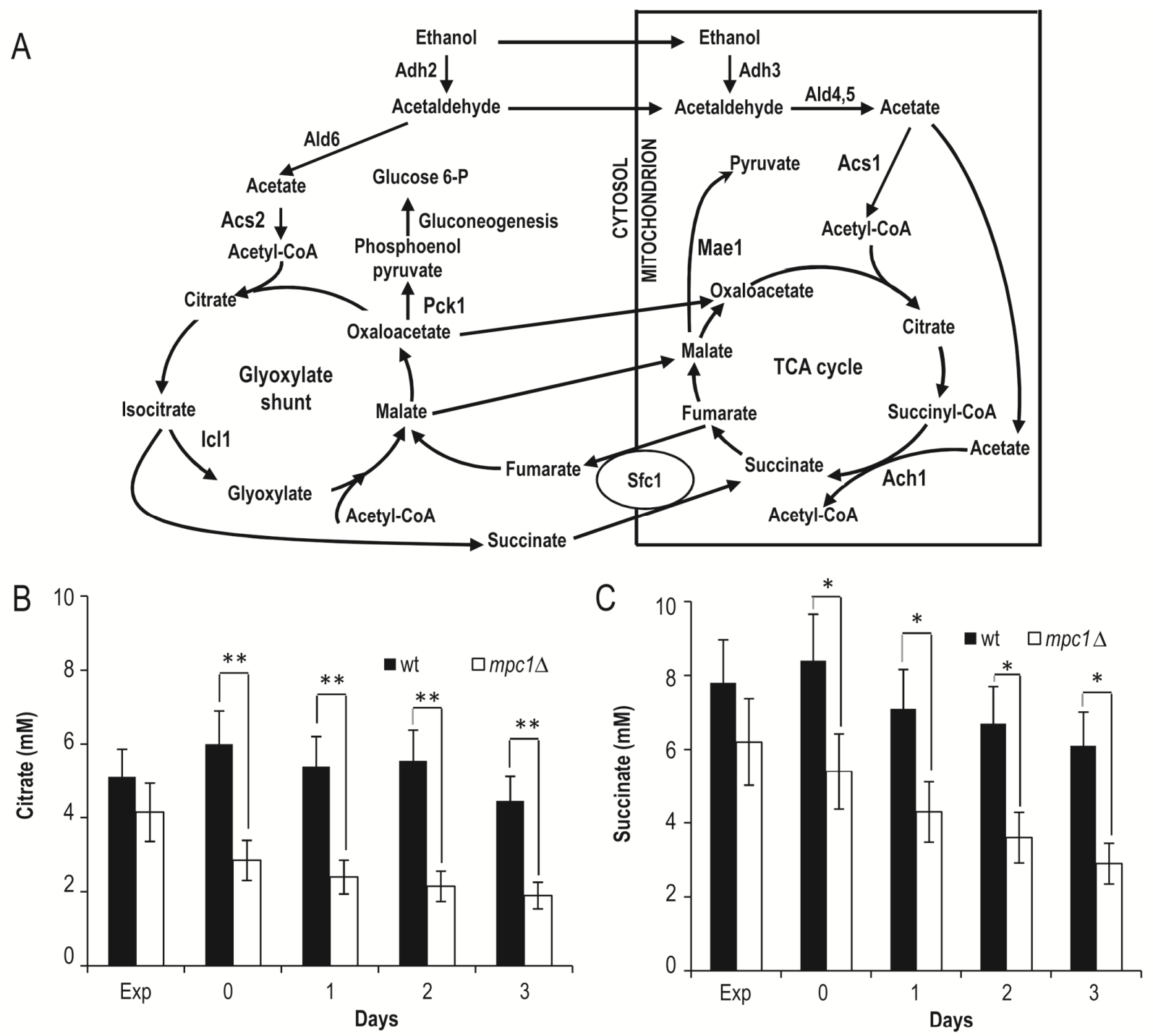

D

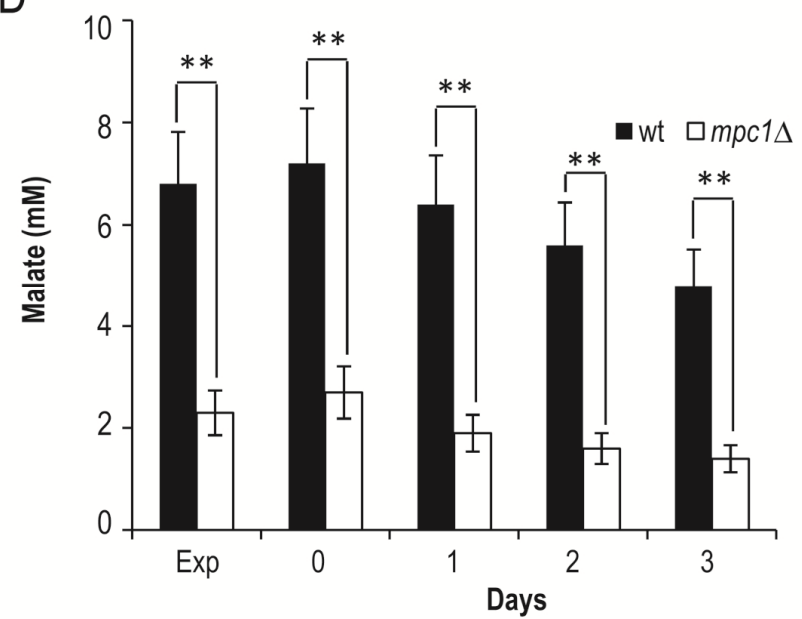

E

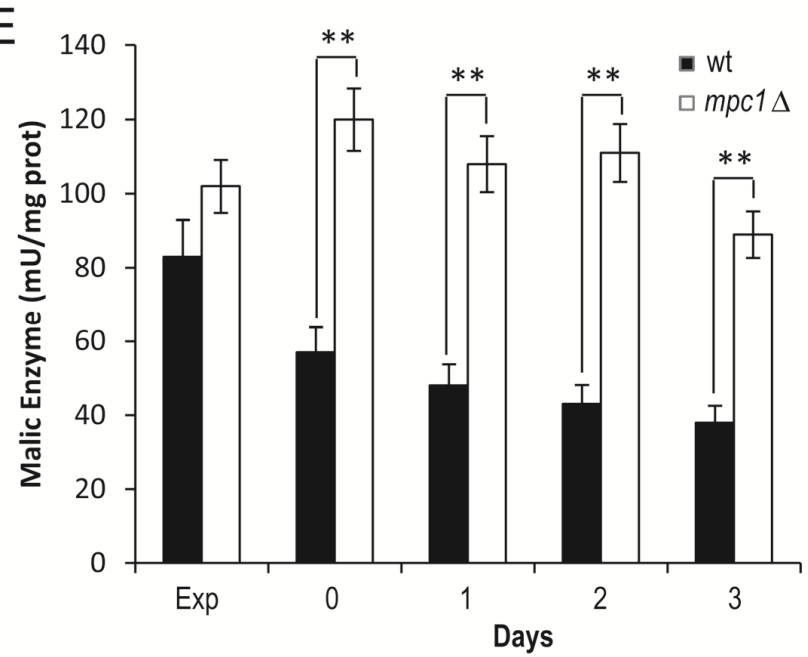

FIGURE 4: Lack of Mpc1 results in low levels of TCA cycle intermediates and enhanced malic enzyme activity. (A) Scheme of the TCA cycle and of the glyoxylate shunt. Ach1, acetyl-CoA hydrolase 1; Acs, acetyl-CoA synthase; Adh, alcohol dehydrogenase; Ald, aldehyde dehydrogenase; Icl1, isocitrate lyase 1; Mae1, malic enzyme; Pck1, phosphoenolpyruvate carboxykinase 1; Sfc1, succinate-fumarate carrier. Wt and $m p c 1 \Delta$ cells were grown as in Figure 2 and at the indicated time points the concentrations of citrate (B), succinate (C) and malate (D) were measured. The bar chart of malic enzyme activity (E) is also reported. Exp, exponential growth phase. Day 0, diauxic shift. Data refer to mean values determined in three independent experiments. SD is indicated. ${ }^{*} P \leq 0.05$ and ${ }^{* *} P \leq 0.01$. 
No significant difference was found between the wt and the $m p c 1 \Delta$ strain in the Adh activity levels in exponential phase (Fig. 3A), where the Adh1 isoenzyme is chiefly responsible for ethanol formation from acetaldehyde, consistent with the similar amounts of ethanol detected in both cultures (Fig. 2B). Similarly, at/after the diauxic-shift where the cytosolic Adh2 is the major ethanol oxidizer, Adh activities displayed no significant difference (Fig. 3A). On the contrary, during all the growth phases analyzed, Ald activity levels were higher in the mutant compared with the wt (Fig. 3B). In particular, a great increase was observed for Ald 6 which is the major cytosolic isoform and is not glucose-repressed [27] compared with that of the mitochondrial counterparts Ald5 and Ald4 (Fig. 3C and D) indicating that the mutant exhibits an increased ability to generate acetate, especially the cytosolic one, which can be used as substrate to produce acetyl-CoA. Accordingly, in the mutant, the nucleocytosolic Acs2-mediated pathway is upregulated during chronological aging [8]. Moreover, an increased cytosolic acetate pool can also account for the extracellular acetate detected in the mpc1s culture (Fig. $2 \mathrm{C})$ whose prolonged accumulation, however, indicates that the flux towards its formation exceeds its utilization. This takes place despite the upregulation of Acs2 enzymatic activity [8] suggesting that the enzyme and/or the flux downstream is/are working at maximum capacity in line with data which show that increase in Acs activity does not result in enhanced acetate utilization $[28,29]$.

Lack of Mpc1 is accompanied by an increase of malic enzyme activity and a decrease in respiration

Starting from these results, we focused on the mitochondrially localized TCA cycle which can be fed with acetyl-CoA generated either from acetate or following oxidation of mitochondrial pyruvate. We measured the levels of citrate, succinate and malate which are intermediates of this cycle but also metabolic connections with the glyoxylate shunt. This is an anaplerotic device of the TCA cycle which allows the formation of $\mathrm{C} 4$ units from $\mathrm{C} 2$ units (acetate) by bypassing oxidative decarboxylation (Fig. 4A) [30]. At/after the diauxic shift, a clear global decrease was observed for all three intermediates in the $m p c 1 \Delta$ cells compared with the wt counterparts (Fig. 4B-D). This decrease was particularly marked for malate which can be used to generate pyruvate in the mitochondria for biosynthetic purposes. This reaction of oxidative decarboxylation is catalyzed by the mitochondrial malic enzyme encoded by MAE1 [31]. As shown in Fig. $4 \mathrm{E}$, in the mpc1 $\triangle$ mutant during the postdiauxic phase, the malic enzyme activity was doubled in comparison with the wt, suggesting that the impairment of pyruvate import into mitochondria linked to Mpc1 loss is compensated by a flux redirection through the Mae1dependent alternative route. This can explain the severe growth defect observed by [22] when the mpc1 $\Delta$ allele was combined with MAE1 deletion.

Moreover, when cells switched to a respiration-based metabolism by using ethanol and acetate, the glyoxylate shunt becomes operative and begins replenishing the TCA cycle intermediates. In addition, during growth on C2 com-
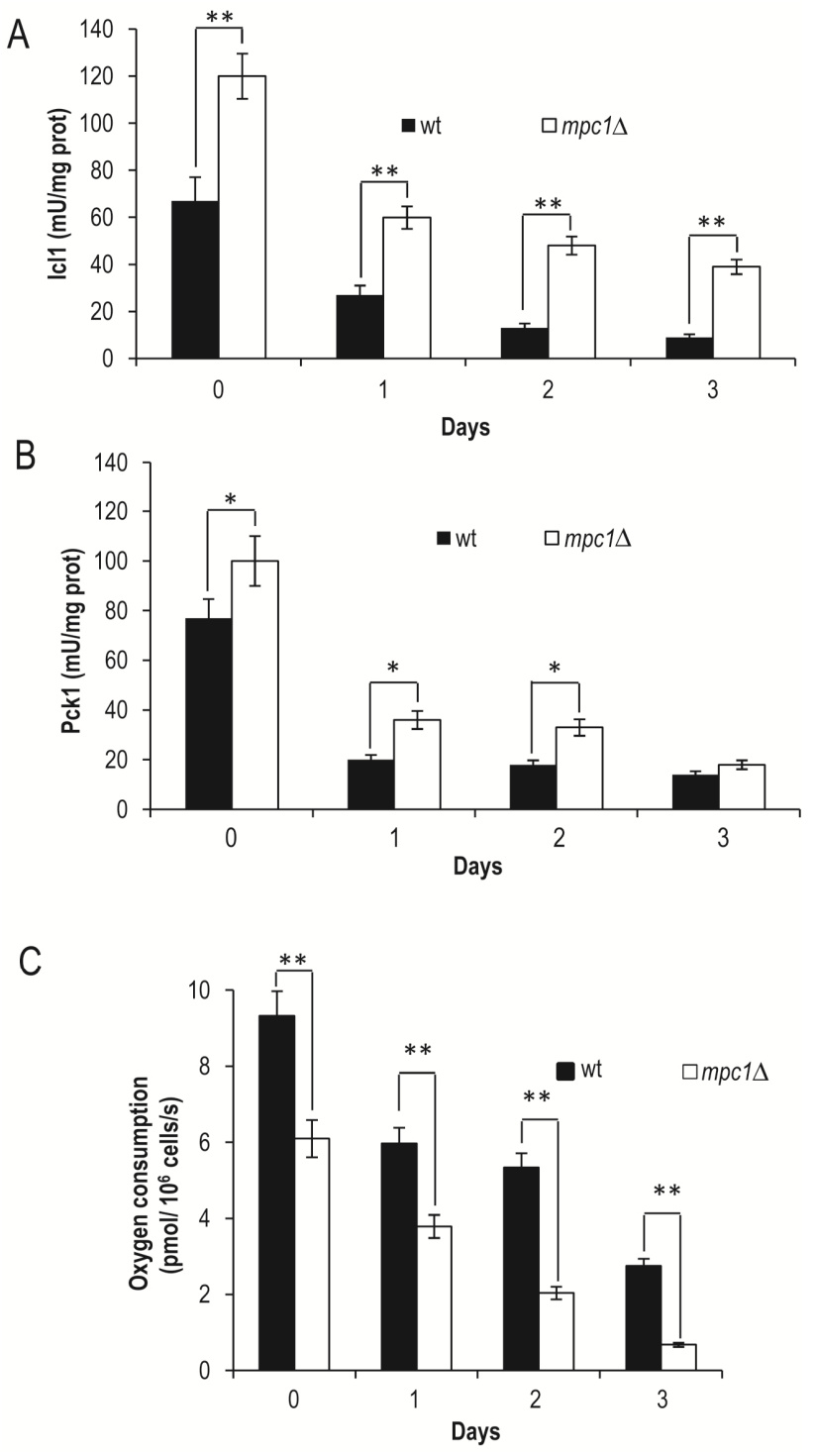

FIGURE 5: MPC1 inactivation increases glyoxylate/gluconeogenesis and reduces respiration during chronological aging. At the indicated time points Icl1 (A) and Pck1 (B) enzymatic activities of wt and mpc1s mutant cells were measured. In parallel, cellular respiration (C) was also monitored. Day 0 , diauxic shift. Data refer to mean values determined in three independent experiments. SD is indicated. ${ }^{*} \mathrm{P} \leq 0.05$ and ${ }^{* *} \mathrm{P} \leq 0.01$.

pounds, this shunt is the exclusive source of oxaloacetate which is the substrate of phosphoenolpyruvate carboxykinase (Pck1), the key enzyme of gluconeogenesis [32]. Measurements of the enzymatic activities of isocitrate lyase (Icl1), which is one of the unique enzymes of the glyoxylate shunt, and Pck1 indicated that these activities were higher in $m p c 1 \Delta$ cells compared with wt ones (Fig. 5A and B). Concomitantly, in mpc1 $\Delta$ cells cellular respiration decreased (Fig. 5C). Icl1 is localized in the cytosol and from isocitrate it generates succinate and the name-giving metabolite glyoxylate which condenses with acetyl-CoA yielding malate. The last one can return to the mitochondria 
(Fig. 4A). Similarly, the major fate of cytosolic succinate is assumed to be its transfer into mitochondria [30]. Moreover, its transport by the Sfc1 carrier provides cytosolic fumarate for conversion to malate which can be used for gluconeogenesis [33]. Thus, taken together, these data indicate that in the mpc1s mutant, an increase in the glyoxylate shunt might represent an increase in metabolite feeding from the cytosol to support a mitochondrial impaired TCA cycle. In this context, the cytosol of the mutant can provide the metabolic environment required to fulfill the increased requirement of substrates for the glyoxylate shunt. In fact, the end-product of the Acs2 synthetase, which is increased in the mutant [8], is the nucleocytosolic acetyl-CoA. In the cytosol, this metabolite, following condensation with oxaloacetate, produces citrate which is then isomerized to isocitrate (the substrate of Icl1). In addition, the cytosol of the mutant might also be a suitable environment which can "promote" Pck1 enzymatic activity. In fact, Pck1 is acetylated by Esa1 and this acetylation is required for its enzymatic activity: an increase of Pck1 en- zymatic activity is associated with an increase of the acetylated form of the enzyme [34, 35]. Accumulating evidence indicates that the availability of acetyl-CoA, the donor substrate for acetylation, can be a metabolic input for the acetylation itself [36-38], so it is reasonable to hypothesize that changes of acetyl-CoA levels may also influence Esa1 activity.

\section{Carnitine restores chronological longevity of the mpc1s mutant}

After the diauxic shift, a metabolic change from fermentation to respiration takes place implying that energy metabolism relies on mitochondrial functionality. Since in the $m p c 1 \Delta$ cells we observed a decrease in respiration, we decided to analyze mitochondrial membrane potential and morphology by using the fluorescent dye, 3,3"dihexyloxacarbocyanine iodide $\left(\mathrm{DiOC}_{6}\right)$ [39]. In fact, mitochondrial morphology reflects the functional status of mitochondria and is regulated by the orchestrated balance of two opposing events: fission and fusion of mitochondria
A

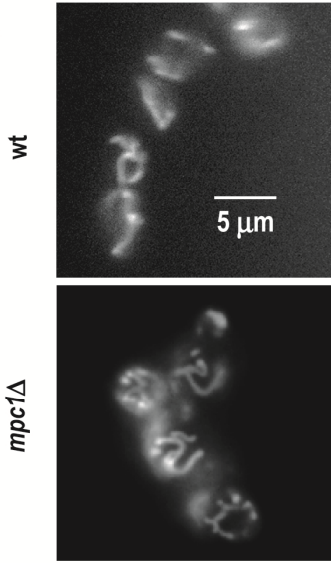

Late exp

C

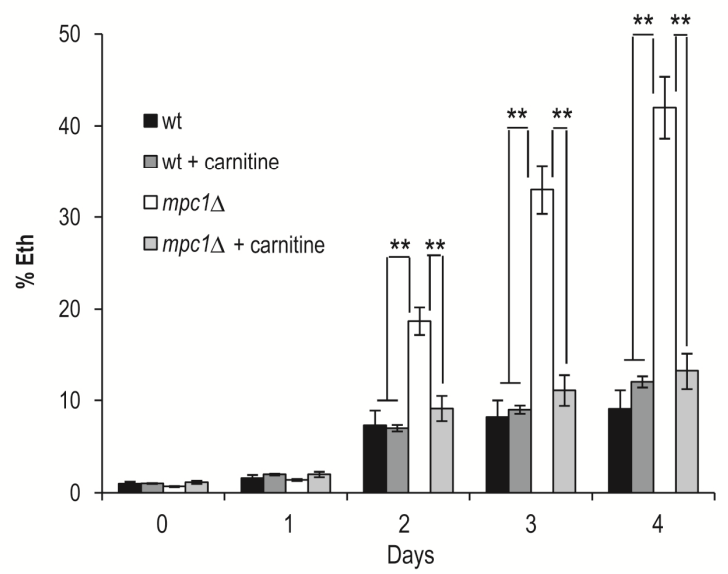

B

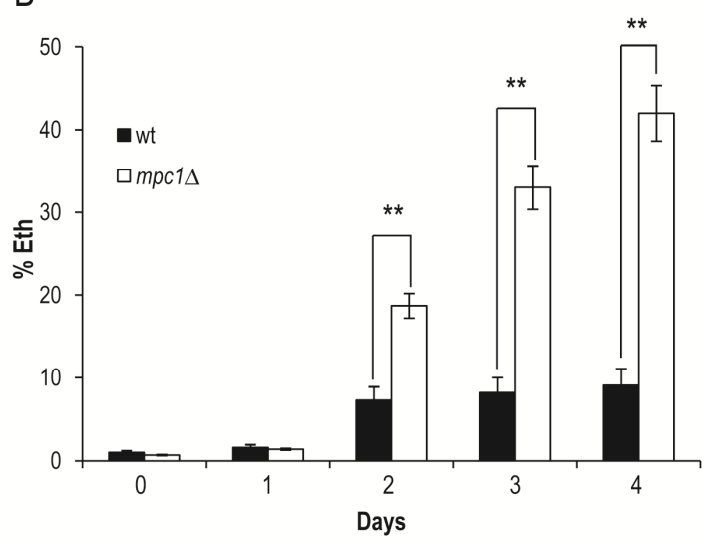

Day 4

D

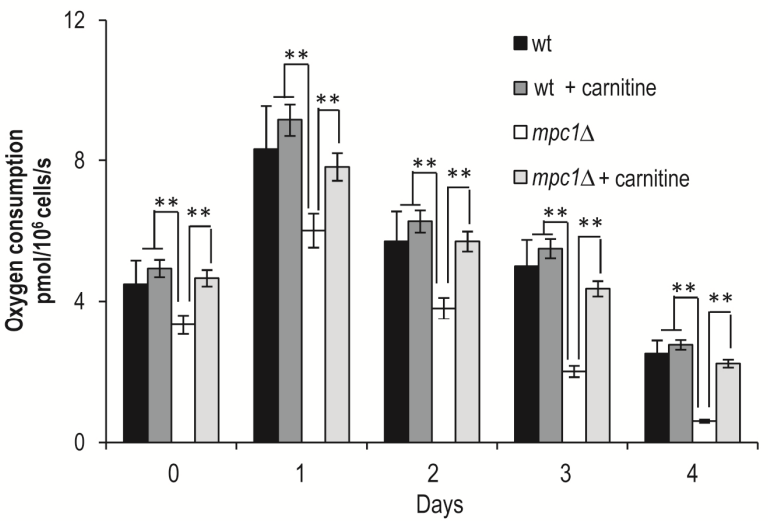

FIGURE 6: Chronologically aging $m p c 1 \Delta$ cells display damaged mitochondria. (A) Representative images of wt and $m p c 1 \Delta$ cultures of Figure 2 stained with $\mathrm{DiOC}_{6}$ to visualize mitochondrial membranes. Morphologies of the mitochondria in late exponential phase (Late exp) are also shown. The same cultures were assessed for the presence of intracellular superoxide by conversion of non-fluorescent dihydroethidium into fluorescent ethidium (Eth). Summary graphs of the percentage of fluorescent/superoxide positive cells (\% Eth) are reported (B). (C) Summary graphs of \% Eth cells and (D) cellular respiration determined in wt and $m p c 1 \Delta$ cultures grown in minimal medium/ $2 \%$ glucose supplemented with carnitine $(10 \mathrm{mg} / \mathrm{L})$. Day 0, diauxic shift. For the determination of Eth cells, evaluation of about 1000 cells for each sample (three technical replicates) in three independent experiments was performed. SD is indicated. ${ }^{*} \mathrm{P} \leq 0.05$ and ${ }^{* *} \mathrm{P} \leq 0.01$. 


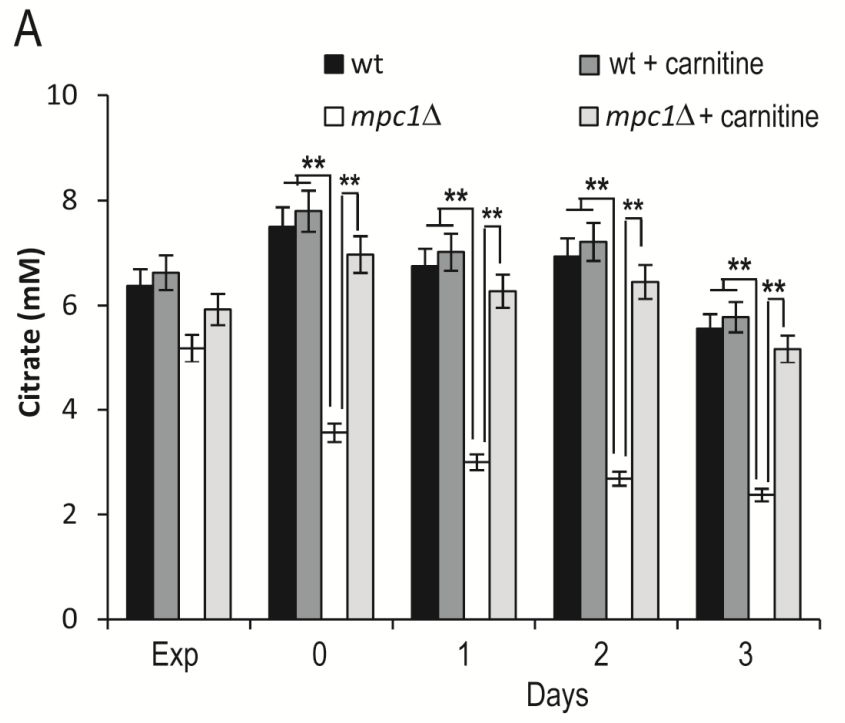

B

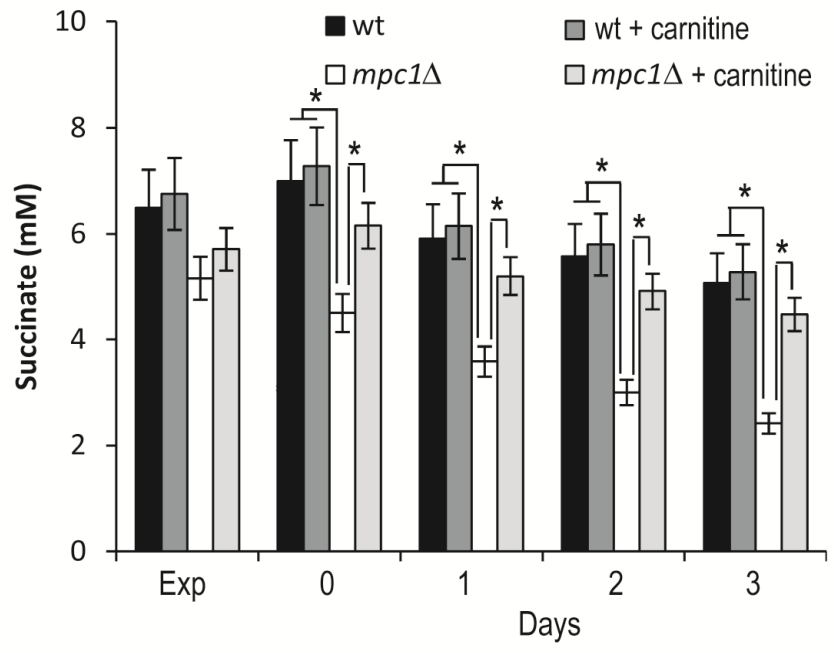

C

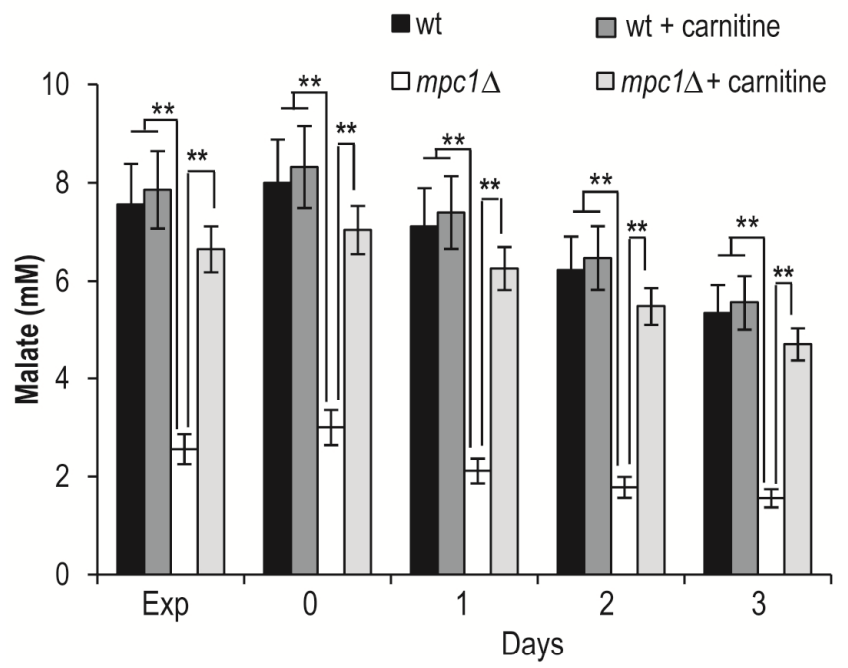

D

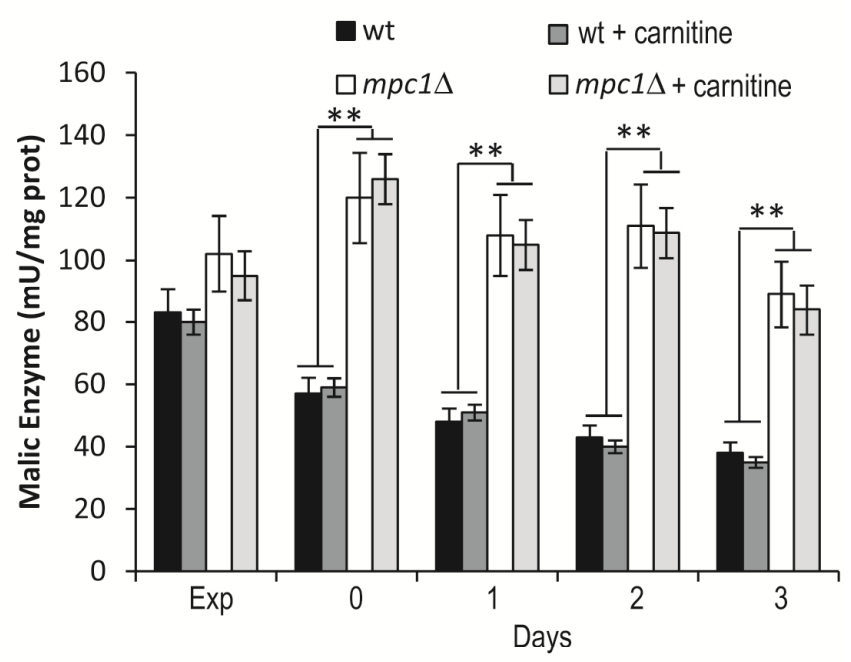

E
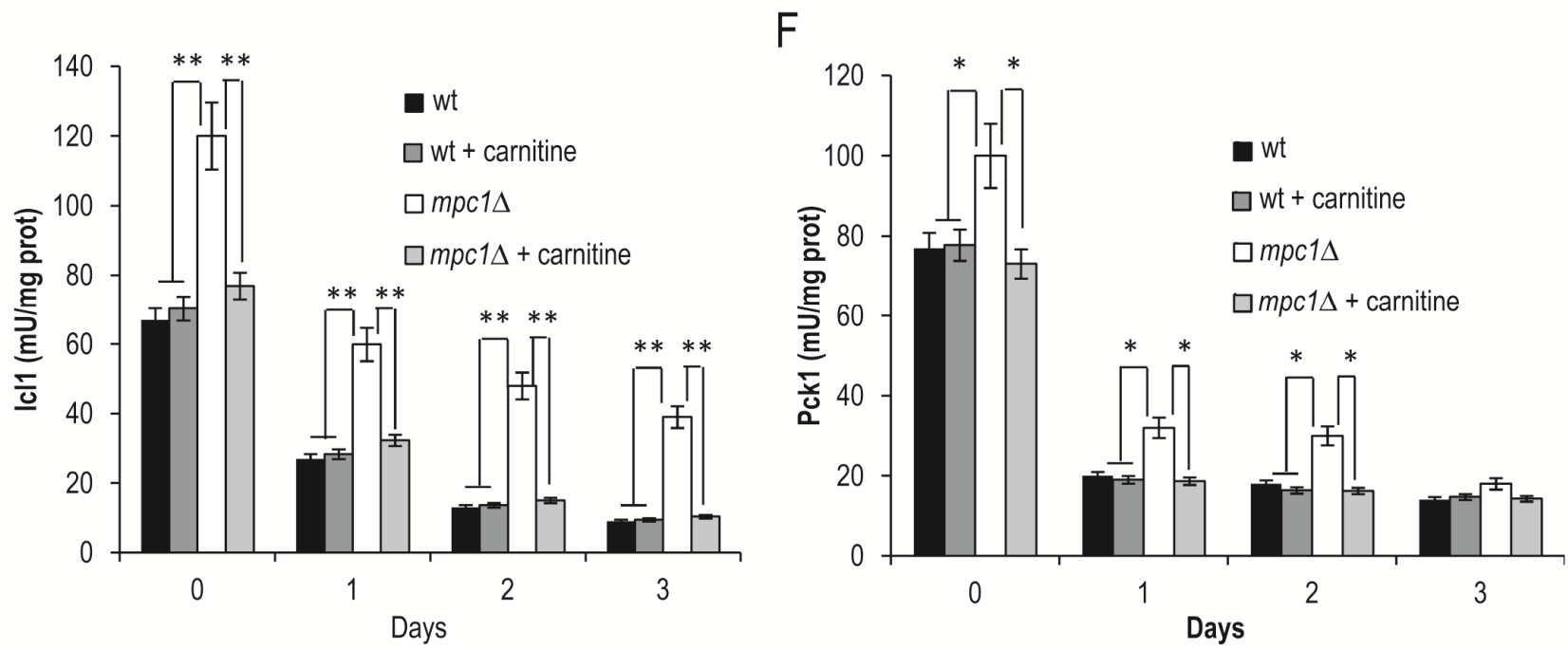

FIGURE 7: Carnitine increases the levels of the TCA cycle intermediates in the mpc1 $\Delta$ mutant. Wt and $m p c 1 \Delta$ cells were grown in minimal medium $/ 2 \%$ glucose supplemented with carnitine $(10 \mathrm{mg} / \mathrm{L})$ and, at the indicated time points, the concentrations of citrate $(A)$, succinate $(B)$ and malate (C) were measured together with Mae1 (D), Icl1 (E) and Pck1 (F) enzymatic activities. Day 0, diauxic shift. Data refer to mean values determined in three independent experiments. SD is indicated. $* P \leq 0.05$ and $* * P \leq 0.01$. 
[40]. As shown in Fig. 6A, a typical tubular network was observed for wt cells whereas for the mutant fluorescent punctiform structures appeared at Day 2 after the diauxic shift. These structures are indicative of mitochondrial fragmentation and are linked to an elevated activity of the mitochondrial fission machinery [41]. In addition to an altered morphology, the mitochondria of the mutant displayed a time-dependent reduction in membrane potential and, at Day 4, did not accumulate $\mathrm{DiOC}_{6}$ (Fig.6A). Mitochondrial dysfunctions are intrinsically related to reactive oxygen species (ROS) of which superoxide anion is one of the most potentially harmful. This radical derives mainly from leakage of electrons from the respiratory chain and, among others, can target mitochondria with detrimental effects $[42,43]$. Chronologically aging $m p c 1 \Delta$ cells had a higher ROS content, measured as the superoxide-driven conversion of non-fluorescent dihydroethidium (DHE) into fluorescent ethidium (Eth), compared with that of the wt cells (Fig. 6B). Notably, culturing $m p c 1 \Delta$ cells in a carnitinesupplemented medium was sufficient to avoid this phenomenon (Fig. 6C). Moreover, oxygen consumption measurements indicated that in these cells cellular respiration increased (Fig. 6D). In S.cerevisiae, carnitine is involved in a process referred to as the carnitine shuttle which allows the transport of acetyl-CoA to the mitochondria. This transport system which is non-functional unless carnitine is supplied with the medium [44, 45], involves the transfer of the acetyl moiety of acetyl-CoA to carnitine and the subsequent transport of the acetylcarnitine to the mitochondria. Here, a mitochondrial carnitine acetyltransferase catalyses the reverse reaction generating carnitine and acetyl-CoA which enters the TCA cycle $[44,46]$. As shown in Fig. 7A-C, the supplemental carnitine did not significantly affect the levels of citrate, succinate and malate in the wt whilst this was not the case for the mpc1 $\Delta$ mutant where the levels of all three intermediates increased and were restored to wtlike ones. No effect was observed on the malic enzyme activity which at/after the post diauxic shift in the $m p c 1 \Delta$ cells was still the double of that of the wt (Fig. 7D) suggesting that the presence of carnitine does not abolish the Mae1-dependent flux towards mitochondrial pyruvate generation. Concomitantly, in the mpc1s cells the enzymatic activities of Icl1 and Pck1 were reduced to the physiological levels measured in the wt (Fig. 7D and E). Thus, all this suggests that in the mpc1 $\Delta$ mutant the activation of the carnitine shuttle can properly feed the TCA cycle by supplying acetyl-CoA to the mitochondria. Hence, the compensative metabolite feeding from the cytosol provided by the glyoxylate shunt seems to be no longer required. Moreover, following carnitine supplementation, during the post-diauxic phase no effect was observed on the ethanol consumption in both the wt and the mutant strains (Fig. $8 \mathrm{~A})$. Similarly, the acetate utilization in the wt was not affected, while in the mpc1 $\Delta$ mutant its utilization was promoted (Fig. 8B). This indicates that in the latter the activation of the carnitine shuttle and the consequent acetyl-CoA transport to the mitochondria can result in an enhancement in the flux downstream from the acetate activation allowing acetate utilization. Finally, in the mpc1 $\Delta$ mutant these metabolic changes matched the almost completely
A

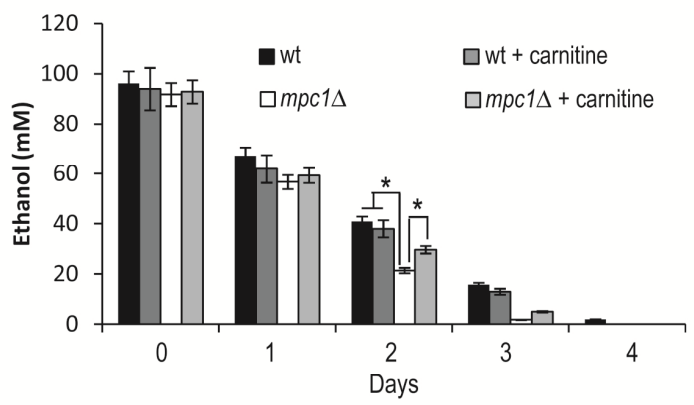

B

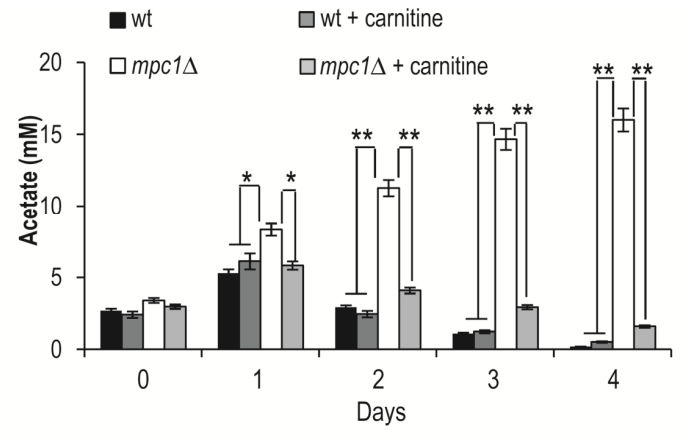

C

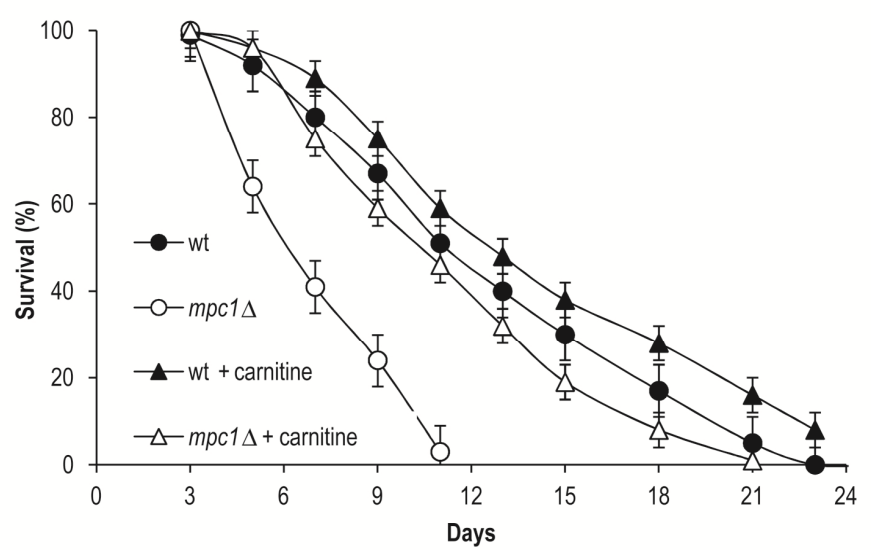

FIGURE 8: Carnitine promotes acetate utilization in the mpc1s mutant in concert with increased CLS. Bar charts of extracellular ethanol (A) and acetate (B) concentrations at different time points after the diauxic shift (Day 0) measured for cells grown as in Figure 7. (C) CLS of the same cells determined as in Figure 2. Data refer to mean values of three independent experiments. $\mathrm{SD}$ is indicated. $* \mathrm{P} \leq 0.05$ and $* * \mathrm{P} \leq 0.01$. 
restored chronological longevity (Fig. 8C).

In conclusion, these data collectively indicate that the lack of the Mpc1 transporter brings about a chain of metabolic events which, in order to counteract the decrease of the pyruvate supply in the mitochondria, by influencing the global acetyl-CoA metabolism ultimately restrict cell survival during chronological aging. In particular, after the diauxic shift when cells utilize the earlier produced ethanol/acetate and increase their respiration demand, one of the metabolic traits of the mpc1 $\Delta$ mutant is a TCA cycle operating in a "branched" fashion with a propensity to shunt intermediates towards pyruvate generation via the malic enzyme. This kind of not-complete cyclic functioning of the TCA cycle by depleting it of intermediates influences not only the respiration, which is reduced in the mutant, but also might reduce mitochondrial acetyl-CoA pool. In fact, a TCA cycle characterized by low levels of intermediates (Fig. 4B-D) generates less succinyl-CoA. This is the substrate for the CoA-transferase's reaction from succinyl-CoA to acetate, catalyzed by Ach1 in cells released from glucose repression [20]. In the mitochondria, this reaction allows the production of acetyl-CoA [8]. Moreover, the CoAtransferase's reaction is using acetate as acceptor, which implies that Ach1 is also important for acetate detoxification and mitochondrial functionality during chronological aging [47]. Consequently, in a condition where acetategenerating activities of Ald4/Ald5 are increased, as it is the case in the mutant, a reduction in CoA-transferase's enzymatic activity could play a causative role in promoting/enhancing the mitochondrial damage observed in the mutant.

Furthermore, since during the utilization of ethanol and acetate, the sole possible route for the net synthesis of C4 dicarboxylic acids for replenishing the TCA cycle of intermediates is the glyoxylate shunt, it follows that in the $m p c 1 \Delta$ cells this anaplerotic shunt is enhanced in order to keep the "branched" TCA cycle functioning. In turn, it follows that the pathway providing the cytosolic acetyl-CoA must be increased to support an enhanced glyoxylate demand. In line with this, the cytosolic Ald 6 enzymatic activity is increased (Fig. 3C) and hyperactivation of the Acs2 activity has been detected [8]. Interestingly, this synthetase is responsible not only for supplying acetyl-CoA for carbon metabolism, but also for protein acetylation, particularly of histones [19]. In addition, it has been shown that during chronological aging, upregulation of Acs2 activity culminates in histone $\mathrm{H} 3$ hyperacetylation associated with transcriptional downregulation of several autophagyessential ATG genes [48]. In this context, mpc1 $\Delta$ cells display an age-dependent loss of autophagy [8]; this feature, given the reciprocal cross-talk between autophagy and mitochondria, can negatively affect the removal of the damaged mitochondria of the mutant and consequently contribute to its inability to maintain proper cellular homeostasis during the aging process. Notably, Ald6, which is responsible of generating cytosolic acetate, is degraded preferentially by autophagy [49] and the persistence of its enzymatic activity seems to be disadvantageous for the survival during nitrogen starvation [50]. Thus, $m p c 1 \Delta$ cells make up for their impairment in mitochondrial pyruvate with a metabolic rewiring in which the pro-aging outcome prevails.

\section{MATERIALS AND METHODS \\ Yeast strains and growth conditions}

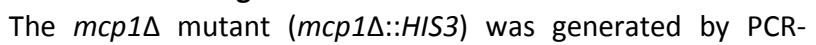
based methods in a BY4741 background (MATa his3 $\Delta-1$ leu2 $\Delta$ 0 met $15 \Delta-0$ ura3 $\Delta-0$ ) and the accuracy of gene replacement was verified by PCR with flanking and internal primers. At least two different clones were tested for any experiment. Yeast cells were grown in batches at $30^{\circ} \mathrm{C}$ in minimal medium (Difco Yeast Nitrogen Base without amino acids, $6.7 \mathrm{~g} / \mathrm{L}$ ) with $2 \%$ glucose and the required supplements added in excess to a final concentration of $200 \mathrm{mg} / \mathrm{L}$, except for leucine at 500 $\mathrm{mg} / \mathrm{L}$ to avoid auxotrophy starvation $[51,52]$. L-carnitine (Sigma) was supplemented to a concentration of $10 \mathrm{mg} / \mathrm{L}$. Strains were inoculated at the same cellular density (culture volume no more than $20 \%$ of the flask volume) and growth was monitored by determining cell number using a Coulter CounterParticle Count and Size Analyser, as described [53]. Duplication times (Td) were obtained by linear regression of the cell number increase over time on a semi-logarithmic plot.

\section{CLS determination}

Survival experiments in expired medium were performed on cells grown in minimal medium $/ 2 \%$ glucose and the required supplements as described above. During growth, cell number and extracellular glucose, ethanol and acetic acid were measured in order to define the growth profile (exponential phase, diauxic shift, post-diauxic phase and stationary phase) of the culture (Fig. S1). Cell survival was monitored by harvesting aliquots of cells starting with $72 \mathrm{~h}$ (Day 3, first age-point) after the diauxic shift (Day 0). CLS was measured according to [51] by counting colony-forming units (CFUs) every 2-3 days. The number of CFUs on Day 3 was considered the initial survival (100\%).

\section{Metabolite measurements and enzymatic assays}

At designated time points, aliquots of the yeast cultures were centrifuged and both pellets (washed twice) and supernatants were frozen at $-80^{\circ} \mathrm{C}$ until used. Rapid sampling for intracellular metabolite measurements was performed according to the leakage-free cold methanol quenching method developed by [54] in which pure methanol at $\leq-40^{\circ} \mathrm{C}$ and a ratio of cell culture to quenching solvent of 1:5 (final methanol concentration $\geq 83 \%$ ) were used. Metabolites from the cell pellets were extracted in $5 \mathrm{ml}$ of a solution of $75 \%(\mathrm{v} / \mathrm{v})$ boiling absolute ethanol containing $0.25 \mathrm{M}$ Hepes, $\mathrm{pH} 7.5$, as described in [55]. The concentrations of glucose, ethanol, acetate, pyruvate, citrate, succinate and malate were determined using enzymatic assays (K-HKGLU, K-ETOH, K-ACET, K-PYRUV, K-SUCC, K-CITR and K-LMALR kits from Megazyme). Ethanol specific consumption rate $(\mathrm{qEtOH})$, expressed in $\mathrm{mmol} \cdot \mathrm{g} \cdot \mathrm{DW}^{-1} \cdot \mathrm{h}^{-1}$, was calculated from measured cell dry weights (DWs) and extracellular ethanol concentrations. DW was measured as described [56].

All the enzymatic activities were assayed immediately after preparation of cell-free extracts. Cells were resuspended in $100 \mathrm{mM}$ potassium phosphate buffer, $\mathrm{pH} 7.5$, containing 2 $\mathrm{mM} \mathrm{MgCl} 2$ and $1 \mathrm{mM}$ dithiothreitol and broken with acidwashed glass beads by shaking on a vortex for several cycles 
interspersed with cooling on ice. The activities of cytosolic and mitochondrial aldehyde dehydrogenase (Ald) were measured as described by [57], of alcohol dehydrogenase (Adh) according to [58], of phosphoenolpyruvate carboxykinase (Pck1) and isocitrate lyase (ICl1) as in [34]. Malic enzyme activities were determined according to [31] with either $0.4 \mathrm{mM} \mathrm{NAD}^{+}$or $\mathrm{NADP}^{+}$as the redox cofactor. The enzymatic activity was measured in the decarboxylation direction to avoid interference with pyruvate decarboxylase and Adh. Total protein concentration was estimated using the $\mathrm{BCA}^{\mathrm{TM}}$ Protein Assay Kit (Pierce).

\section{Oxygen consumption and fluorescence microscopy}

The basal oxygen consumption of intact cells was measured at $30^{\circ} \mathrm{C}$ using a 'Clark-type' oxygen electrode in a thermostatically controlled chamber (Oxygraph System, Hansatech Instruments, Norfolk, UK) as previously reported [25]. Data were recorded at sampling intervals of $1 \mathrm{~s}$ (Oxygraph Plus software, Hansatech Instruments, Norfolk, UK). All assays were conducted in biological triplicate.

ROS were detected with dihydroethidium (DHE, Sigma) according to [59]. The mitochondrial membrane potential was assessed by staining with $\mathrm{DiOC}_{6}$ (Molecular Probes, Invitrogen), according to [39]; cells were also counterstained with propidium iodide to discriminate between live and dead cells. A Nikon Eclipse E600 fluorescence microscope equipped with a Leica DC 350F ccd camera was used. Digital images were acquired using FW4000 software (Leica).

\section{Statistical analysis of data}

All values are presented as the mean of three independent experiments with the corresponding Standard Deviation (SD).

\section{REFERENCES}

1. Longo VD, Kennedy BK (2006). Sirtuins in aging and age-related disease. Cell 126(2): 257-68.

2. MacLean M, Harris N, Piper PW (2001). Chronological lifespan of stationary phase yeast cells; a model for investigating the factors that might influence the ageing of postmitotic tissues in higher organisms. Yeast 18(6): 499-509.

3. Fabrizio P, Longo VD (2003). The chronological life span of Saccharomyces cerevisiae. Aging Cell 2(2): 73-81.

4. Fontana L, Partridge L, Longo VD (2010). Extending healthy life span - from yeast to humans. Science 328(5976): 321-6.

5. Swinnen E, Ghillebert R, Wilms T, Winderickx J (2013). Molecular mechanisms linking the evolutionary conserved TORC1-Sch9 nutrient signalling branch to lifespan regulation in Saccharomyces cerevisiae. FEMS Yeast Res 14(1): 17-32.

6. Houtkooper RH, Williams RW, Auwerx J (2010). Metabolic networks of longevity. Cell 142(1): 9-14.

7. de Cabo R, Carmona-Gutierrez D, Bernier M, Hall MN, Madeo F (2014). The search for antiaging interventions: from elixirs to fasting regimens. Cell 157(7): 1515-26.
Three technical replicates were analyzed in each independent experiments. Statistical significance was assessed by one-way ANOVA test. $P$ value of $\leq 0.05$ was considered statistically significant.

\section{ACKNOWLEDGMENTS}

The authors are grateful to Neil Campbell for English editing. We acknowledge funding of the project SysBioNet, Italian Roadmap Research Infrastructures.

\section{SUPPLEMENTAL MATERIAL}

All supplemental data for this article are available online at www.microbialcell.com.

\section{CONFLICT OF INTEREST}

The authors declare no conflict of interest.

\section{COPYRIGHT}

(C) 2014 Orlandi et al. This is an open-access article released under the terms of the Creative Commons Attribution (CC BY) license, which allows the unrestricted use, distribution, and reproduction in any medium, provided the original author and source are acknowledged.

Please cite this article as: Ivan Orlandi, Damiano Pellegrino Coppola and Marina Vai (2014). Rewiring yeast acetate metabolism through MPC1 loss of function leads to mitochondrial damage and decreases chronological lifespan. Microbial Cell 1(12): 393-405. doi: 10.15698/mic2014.12.178

8. Eisenberg $T$, Schroeder $S$, Andryushkova A, Pendl $T$, Kuttner $\mathrm{V}$, Bhukel A, Mariño G, Pietrocola F, Harger A, Zimmermann A, Moustafa $T$, Sprenger A, Jany E, Buttner S, Carmona-Gutierrez D, Ruckenstuhl C, Ring J, Reichelt W, Schimmel K, Leeb T, Moser C, Schatz S, Kamolz LP, Magnes C, Sinner F, Sedej S, Frohlich KU, Juhasz G, Pieber TR, Dengjel J, Sigrist SJ, Kroemer G, Madeo F (2014). Nucleocytosolic depletion of the energy metabolite acetyl-coenzyme A stimulates autophagy and prolongs lifespan. Cell Metab 19(3): 431-44.

9. Friis RM, Glaves JP, Huan T, Li L, Sykes BD, Schultz MC (2014). Rewiring AMPK and mitochondrial retrograde signaling for metabolic control of aging and histone acetylation in respiratory-defective cells. Cell $\operatorname{Rep} 7(2)$ : 565-74.

10. Mariño G, Pietrocola $F$, Eisenberg T, Kong $Y$, Malik SA, Andryushkova A, Schroeder S, Pendl T, Harger A, Niso-Santano M, Zamzami N, Scoazec M, Durand S, Enot DP, Fernandez AF, Martins I, Kepp O, Senovilla L, Bauvy C, Morselli E, Vacchelli E, Bennetzen M, Magnes C, Sinner F, Pieber T, Lopez-Otin C, Maiuri MC, Codogno P, Andersen JS, Hill JA, Madeo F, Kroemer G (2014). Regulation of autophagy by cytosolic acetyl-coenzyme A. Mol Cell 53(5): 710-25.

11. Henriksen $P$, Wagner SA, Weinert BT, Sharma S, Bacinskaja G, Rehman M, Juffer AH, Walther TC, Lisby M, Choudhary C (2012). Proteome-wide analysis of lysine acetylation suggests its broad regulatory scope in Saccharomyces cerevisiae. Mol Cell Proteomics 11(11): 151022.

12. Kaelin WG Jr., McKnight SL (2013). Influence of metabolism on epigenetics and disease. Cell 153(1): 56-69. 
13. Lu C, Thompson CB (2012). Metabolic regulation of epigenetics. Cell Metab 16(1): 9-17.

14. Millar CB, Grunstein M (2006). Genome-wide patterns of histone modifications in yeast. Nat Rev Mol Cell Biol 7(9): 657-66.

15. Eisenberg T, Knauer H, Schauer A, Buttner S, Ruckenstuhl C, Carmona-Gutierrez D, Ring J, Schroeder S, Magnes C, Antonacci L, Fussi $H_{\text {, }}$ Deszcz L, Hartl R, Schraml E, Criollo A, Megalou E, Weiskopf D, Laun P, Heeren G, Breitenbach M, Grubeck-Loebenstein B, Herker E, Fahrenkrog B, Frohlich KU, Sinner F, Tavernarakis N, Minois N, Kroemer $G$, Madeo $F$ (2009). Induction of autophagy by spermidine promotes longevity. Nat Cell Biol 11(11): 1305-14.

16. Rubinsztein DC, Mariño G, Kroemer G (2011). Autophagy and aging. Cell 146(5): 682-95.

17. Cuervo AM (2008). Autophagy and aging: keeping that old broom working. Trends Genet 24(12): 604-12.

18. van den Berg MA, de Jong-Gubbels $P$, Kortland $C J$, van Dijken JP, Pronk JT, Steensma HY (1996). The two acetyl-coenzyme A synthetases of Saccharomyces cerevisiae differ with respect to kinetic properties and transcriptional regulation. J Biol Chem 271(46): 28953-9.

19. Takahashi H, McCaffery JM, Irizarry RA, Boeke JD (2006). Nucleocytosolic acetyl-coenzyme $A$ synthetase is required for histone acetylation and global transcription. Mol Cell 23(2): 207-17.

20. Fleck CB, Brock M (2009). Re-characterisation of Saccharomyces cerevisiae Ach1p: fungal CoA-transferases are involved in acetic acid detoxification. Fungal Genet Biol 46(6-7): 473-85.

21. Pronk JT, Yde Steensma H, Van Dijken JP (1996). Pyruvate metabolism in Saccharomyces cerevisiae. Yeast 12(16): 1607-33.

22. Bricker DK, Taylor EB, Schell JC, Orsak T, Boutron A, Chen YC, Cox JE, Cardon CM, Van Vranken JG, Dephoure N, Redin C, Boudina S, Gygi SP, Brivet M, Thummel CS, Rutter J (2012). A mitochondrial pyruvate carrier required for pyruvate uptake in yeast, Drosophila, and humans. Science 337(6090): 96-100.

23. Herzig S, Raemy E, Montessuit S, Veuthey JL, Zamboni N, Westermann B, Kunji ER, Martinou JC (2012). Identification and functional expression of the mitochondrial pyruvate carrier. Science 337(6090): 93-6.

24. Casal M, Paiva S, Queiros O, Soares-Silva I (2008). Transport of carboxylic acids in yeasts. FEMS Microbiol Rev 32(6): 974-94.

25. Orlandi I, Ronzulli R, Casatta N, Vai M (2013). Ethanol and acetate acting as carbon/energy sources negatively affect yeast chronological aging. Oxid Med Cell Longev Article ID:802870.

26. de Smidt O, du Preez JC, Albertyn J (2008). The alcohol dehydrogenases of Saccharomyces cerevisiae: a comprehensive review. FEMS Yeast Res 8(7): 967-78.

27. Saint-Prix F, Bonquist L, Dequin S (2004). Functional analysis of the ALD gene family of Saccharomyces cerevisiae during anaerobic growth on glucose: the NADP+-dependent Ald6p and Ald5p isoforms play a major role in acetate formation. Microbiology 150(7): 2209-20.

28. de Jong-Gubbels $P$, van den Berg MA, Luttik MA, Steensma HY, van Dijken JP, Pronk JT (1998). Overproduction of acetyl-coenzyme A synthetase isoenzymes in respiring Saccharomyces cerevisiae cells does not reduce acetate production after exposure to glucose excess. FEMS Microbiol Lett 165(1): 15-20.

29. Remize F, Andrieu E, Dequin S (2000). Engineering of the pyruvate dehydrogenase bypass in Saccharomyces cerevisiae: role of the cytosolic $\mathrm{Mg}\left(2^{+}\right)$and mitochondrial $\mathrm{K}\left(^{+}\right)$acetaldehyde dehydrogenases Ald6 $p$ and Ald $4 p$ in acetate formation during alcoholic fermentation. Appl Environ Microbiol 66(8): 3151-9.
30. Lee YJ, Jang JW, Kim KJ, Maeng PJ (2011). TCA cycle-independent acetate metabolism via the glyoxylate cycle in Saccharomyces cerevisiae. Yeast 28(2): 153-66.

31. Boles $E$, de Jong-Gubbels $P$, Pronk JT (1998). Identification and characterization of MAE1, the Saccharomyces cerevisiae structural gene encoding mitochondrial malic enzyme. J Bacteriol 180(11): 287582.

32. dos Santos MM, Gombert AK, Christensen B, Olsson L, Nielsen J (2003). Identification of in vivo enzyme activities in the cometabolism of glucose and acetate by Saccharomyces cerevisiae by using ${ }^{13} \mathrm{C}$ labeled substrates. Eukaryot Cell 2(3): 599-608.

33. Palmieri L, Lasorsa FM, De Palma A, Palmieri F, Runswick MJ, Walker JE (1997). Identification of the yeast ACR1 gene product as a succinate-fumarate transporter essential for growth on ethanol or acetate. FEBS Lett 417(1): 114-8.

34. Casatta N, Porro A, Orlandi I, Brambilla L, Vai M (2013). Lack of Sir2 increases acetate consumption and decreases extracellular pro-aging factors. Biochim Biophys Acta 1833(3): 593-601.

35. Lin YY, Lu JY, Zhang J, Walter W, Dang W, Wan J, Tao SC, Qian J, Zhao Y, Boeke JD, Berger SL, Zhu H (2009). Protein acetylation microarray reveals that NuA4 controls key metabolic target regulating gluconeogenesis. Cell 136(6): 1073-84.

36. Guan KL, Xiong Y (2011). Regulation of intermediary metabolism by protein acetylation. Trends Biochem Sci 36(2): 108-16.

37. Choudhary C, Weinert BT, Nishida Y, Verdin E, Mann M (2014). The growing landscape of lysine acetylation links metabolism and cell signalling. Nat Rev Mol Cell Biol 15(8): 536-50.

38. Cai L, Sutter BM, Li B, Tu BP (2011). Acetyl-CoA induces cell growth and proliferation by promoting the acetylation of histones at growth genes. Mol Cell 42(4): 426-37.

39. Koning AJ, Lum PY, Williams JM, Wright R (1993). DiOC 6 staining reveals organelle structure and dynamics in living yeast cells. Cell Motil Cytoskel 25(2): 111-28.

40. Knorre DA, Popadin KY, Sokolov SS, Severin FF (2013). Roles of mitochondrial dynamics under stressful and normal conditions in yeast cells. Oxid Med Cell Longev Article ID:139491.

41. Merz S, Hammermeister M, Altmann K, Durr M, Westermann B (2007). Molecular machinery of mitochondrial dynamics in yeast. Biol Chem 388(9): 917-26.

42. Breitenbach M, Rinnerthaler M, Hartl J, Stincone A, Vowinckel J, Breitenbach-Koller $H$, Ralser M (2014). Mitochondria in ageing: there is metabolism beyond the ROS. FEMS Yeast Res 14(1): 198-212.

43. Demir AB, Koc A (2010). Assessment of chronological lifespan dependent molecular damages in yeast lacking mitochondrial antioxidant genes. Biochem Biophys Res Commun 400(1): 106-10.

44. van Roermund CW, Hettema EH, van den Berg M, Tabak HF, Wanders RJ (1999). Molecular characterization of carnitine-dependent transport of acetyl-CoA from peroxisomes to mitochondria in Saccharomyces cerevisiae and identification of a plasma membrane carnitine transporter, Agp2p. EMBO J 18(21): 5843-52.

45. Swiegers JH, Dippenaar N, Pretorius IS, Bauer FF (2001). Carnitinedependent metabolic activities in Saccharomyces cerevisiae: three carnitine acetyltransferases are essential in a carnitine-dependent strain. Yeast 18(7): 585-95.

46. Palmieri L, Lasorsa FM, lacobazzi V, Runswick MJ, Palmieri F, Walker JE (1999). Identification of the mitochondrial carnitine carrier in Saccharomyces cerevisiae. FEBS Lett 462(3): 472-6. 
47. Orlandi I, Casatta N, Vai M (2012). Lack of Ach1 coa-transferase triggers apoptosis and decreases chronological lifespan in yeast. Front Oncol 2: 67.

48. Eisenberg T, Schroeder S, Buttner S, Carmona-Gutierrez D, Pendl T, Andryushkova A, Mariño $G$, Pietrocola $F$, Harger A, Zimmermann A, Magnes C, Sinner F, Sedej S, Pieber TR, Dengjel J, Sigrist S, Kroemer G, Madeo $F(2014)$. A histone point mutation that switches on autophagy. Autophagy 10(6): 1143-45.

49. Suzuki K, Nakamura S, Morimoto M, Fujii K, Noda NN, Inagaki F, Ohsumi $Y$ (2014). Proteomic profiling of autophagosome cargo in Saccharomyces cerevisiae. PLoS One 9(3): e91651.

50. Onodera J, Ohsumi Y (2004). Ald6p is a preferred target for autophagy in yeast, Saccharomyces cerevisiae. J Biol Chem 279(16): 16071-6.

51. Fabrizio P, Gattazzo C, Battistella L, Wei M, Cheng C, McGrew K, Longo VD (2005). Sir2 blocks extreme life-span extension. Cell 123(4): 655-67.

52. Boer VM, Amini S, Botstein D (2008). Influence of genotype and nutrition on survival and metabolism of starving yeast. Proc Natl Acad Sci USA 105(19): 6930-5.

53. Vanoni M, Vai M, Popolo L, Alberghina L (1983). Structural heterogeneity in populations of the budding yeast Saccharomyces cerevisiae. J Bacteriol 156(3): 1282-91.
54. Canelas A, Ras C, ten Pierick A, van Dam J, Heijnen J, van Gulik W (2008). Leakage-free rapid quenching technique for yeast metabolomics. Metabolomics 4(3): 226-39.

55. Canelas AB, ten Pierick A, Ras C, Seifar RM, van Dam JC, van Gulik WM, Heijnen JJ (2009). Quantitative evaluation of intracellular metabolite extraction techniques for yeast metabolomics. Anal Chem 81(17): 7379-89.

56. Agrimi G, Brambilla L, Frascotti G, Pisano I, Porro D, Vai M, Palmieri $L$ (2011). Deletion or overexpression of mitochondrial $N^{\prime} A D^{+}$carriers in Saccharomyces cerevisiae alters cellular NAD and ATP contents and affects mitochondrial metabolism and the rate of glycolysis. Appl Environ Microbiol 77(7): 2239-46.

57. Aranda A, del Olmo M (2003). Response to acetaldehyde stress in the yeast Saccharomyces cerevisiae involves a strain-dependent regulation of several $A L D$ genes and is mediated by the general stress response pathway. Yeast 20(8): 747-59.

58. Postma E, Verduyn C, Scheffers WA, Van Dijken JP (1989). Enzymic analysis of the crabtree effect in glucose-limited chemostat cultures of Saccharomyces cerevisiae. Appl Environ Microbiol 55(2): 468-77.

59. Madeo F, Frohlich E, Ligr M, Grey M, Sigrist SJ, Wolf DH, Frohlich KU (1999). Oxygen stress: a regulator of apoptosis in yeast. J Cell Biol 145(4): 757-67. 